\title{
Relationship of in Vitro Acrosome Reaction to Sperm Function: An Update
}

\author{
Sandro C. Esteves* and Sidney Verza Jr.
}

ANDROFERT, Andrology and Human Reproduction Clinic, Campinas, São Paulo, Brazil

\begin{abstract}
Understanding the mechanisms by which the acrosome reaction is regulated is central to models of fertilization. This article reviews the relationship of the acrosome reaction detected in vitro to sperm function. Proteolytic enzymes in the acrosome digest through the zona pellucida allowing for sperm-oolemma fusion. When this process is impaired, either by lack of an acrosome or acrosomal dysfunction, fertility can be compromised. Optical microscopy and staining with different fluorescent lectins that bind to acrosomal membranes is the method of choice for acrosomal evaluation. Because acrosomal loss can be a result of sperm death, this test should be used in in conjunction with an assay to monitor sperm viability. Different stimulants, such as phosphodiesterase inhibitors, drugs and toxins have been investigated in their ability to affect the sperm ability to undergo in vitro acrosome reaction. Sperm acrosomes are also sensitive to the freezing-thawing process and strategies have been described to minimize cryodamage. The assessment of the acrosome has been shown to be a stable parameter of sperm function and a valid tool to predict the fertilizing potential of human spermatozoa. The acrosome reaction following ionophore challenge (ARIC) is an in vitro assay with good predictability of the sperm's fertilizing potential for assisted conception techniques including intrauterine insemination and conventional in vitro fertilization. The AR determination has been also used as an important biomarker in studies involving drugs and toxins. Recently, novel aspects of sperm-oocyte fusion have been depicted in humans involving glycoproteins present in the zona pellucida and the female reproductive tract.
\end{abstract}

Keywords: Spermatozoa, acrosome reaction, infertility, male, cryopreservation, phosphodiesterase inhibitors.

\section{INTRODUCTION}

\section{Human Sperm Head Formation}

The mammalian sperm head is composed of two main structures, the acrosome and the nucleus. The human acrosome is a membrane-bound organelle derived from the Golgi complex. The acrosome formation occurs during spermiogenesis, specifically during the round spermatid stage when accumulation of acrosomal materials and the flattening of acrosome take place [1]. The acrosome is located in the anterior portion of the sperm head and its area varies by species. In humans, the acrosome occupies $40 \%$ to $70 \%$ of the sperm head [2]. Acrosomal contents are composed of proteases, mainly proacrosin, hyaluronidase and phospholipase A2. These substances are released during the early stages of sperm-egg interaction through an exocytotic process known as the acrosome reaction [3].

\section{Capacitation and Acrosome Reaction}

In vivo, ejaculated spermatozoa from all eutherian mammals are unable to fertilize until they have undergone capacitation that allow the acrosome reaction to take place when they approach or contact the oocyte [4]. Capacitation has been recognized as a time-dependent phenomenon, with the absolute time course being species-specific [5]. It prepares the sperm to undergo the acrosome reaction with

*Address correspondence to this author at the ANDROFERT, Av. Dr. Heitor Penteado, 1464, 13075-460 - Campinas - São Paulo, Brazil; Tel: +55 193295-8877; Fax: +55 193294-6992; E-mail: s.esteves@androfert.com.br the accompanying release of lytic enzymes and exposure of membrane receptors, which are required for sperm penetration through the zona pellucida and for fusion with the oolema [5]. Sperm transport through the female genital tract can occur quite rapidly (times as short as 15 to 30 minutes have been reported in humans), whereas the capacitation process can take from 3 to 24 hours [5]. It is speculated, therefore, that capacitation is not completed until after the spermatozoa has entered the cumulus oophorus. This delay is physiologically beneficial because the spermatozoa do not respond to acrosome-reaction-inducing signals until they approach the zona pellucida, preventing premature acrosome reactions that lead ultimately to the sperm inability to penetrate the egg vestments $[5,6]$. Sperm capacitation is a postejaculatory modification of the sperm surface, which involves the mobilization and/or removal of certain surface components from the sperm plasma membrane, such as glycoproteins, decapacitation factor, acrosome-stabilizing factor, and acrosin inhibitor. Sperm capacitation involves major biochemical and biophysical changes in the membrane complex and energy metabolism. The presence of high concentrations of cholesterol in the seminal plasma, which maintains a high cholesterol concentration in sperm membranes, may be the most important factor for the inhibition of capacitation [7]. Capacitation is associated to increased membrane fluidity caused by the removal of cholesterol from sperm plasma membrane via sterol acceptors present in the female tract secretions [8,9]. A marked change in sperm motility, named hyperactivation, is also associated with capacitation. Hyperactivated spermatozoa exhibit an extremely vigorous but nonprogressive motility pattern, as a result of a $\mathrm{Ca}^{2+}$ influx, that causes increased flagellar curva- 
ture [10] and hence extreme lateral movement of the sperm head [8]. Proteasome participates in activating calcium channels that also leads to increased membrane fluidity and permeability [11-14]. These events are followed by or occur simultaneous with [i] a decrease in net surface charge, [ii] devoided area of intra-membrane protein and sterols, and [iii] increased concentrations of anionic phospholipids [14, 15]. Hyperactivated motility is essential for sperm penetration into the intact oocyte-cumulus complexes in vitro and in vivo $[16,17]$.

The spermatozoon binds to the zona pellucida (ZP) with its intact plasma membrane after penetrating into the cumulus oophorous. Sperm binding occurs via specific receptors to ZP glycoproteins located over the anterior sperm head [18]. Glycosylation of ZP glycoproteins are important in sperm-ZP interaction. It is believed that human ZP glycoprotein-3 (ZP3) has a central role in initiating the acrosome reaction [19]; however, it has been recently demonstrated that human ZP1 and ZP4 are also implicated in the process $[20,21]$. The acrosome reaction is a stimulus-secretion coupled exocytotic event in which the acrosome fuses with the overlying plasma membrane $[22,23]$. The multiple fusions between the outer acrosomal membrane and the plasma membrane result in the release of hydrolytic enzymes (mostly acrosin) and in the exposure of new membrane domains, both of which are essential if fertilization is to proceed further. The hydrolytic enzymes released from the acrosome digest the zona pellucida, allowing the spermatozoa to penetrate the oocyte [18]. The acrosome reaction (AR) seems to be physiologically induced by natural stimulants such as the follicular fluid (FF), progestin, progesterone, and hydroxy progesterone [15]. Follicular fluid and cumulus cells have protein-bound progesterone that has been identified as one of the most important acrosome reaction-inducing agents [23, 24]. Follicular fluid stimulates the acrosome reaction in a dose-dependent manner [24, 25]. A link between locally produced estradiol by ejaculated spermatozoa, acrosome reaction and sperm capacitation has been also described [26]. Moreover, evidence indicates that environmental estrogens can significantly stimulate mammalian sperm capacitation and acrosome reaction [27]. The acrosome reaction is probably initiated when ligands produced by the oocyte bind to the receptors on the spermatozoa. This signal is transduced intracellularly via second messengers, ultimately leading to exocytosis [28]. A number of second messenger pathways have been identified in human spermatozoa, including those that result in the activation of cyclic adenosine monophosphate (cAMP), cyclic guanosine monophosphate (cGMP), and phospholipid dependent protein kinases [14, 29-31]. These kinases are called, respectively, protein kinase $A$, protein kinase $\mathrm{G}$, and protein kinase C. It is possible that these pathways interact to assure an optimal response at the correct place and time during the fertilization process. Although the concentration of cyclic GMP in ejaculated human semen is almost 7 times lower than cAMP, it is speculated that both nucleotides have a similar role in the AR since their dependent protein kinases are closely related proteins [32-34]. The AR can also be induced by artificial stimulants that cause and increase in sperm intracellular calcium $[14,35]$.

\section{Importance of the Acrosome Reaction in Vivo and in Vitro}

Acrosome integrity is crucial for normal fertilization both in vivo and in vitro. A high proportion of sperm with intact acrosomes is seen in ejaculates of normal men. In such individuals, $\sim 5-20 \%$ sperm cells may exhibit spontaneous acrosome reactions that are of no clinical significance [35]. Conversely, several abnormal conditions affecting the sperm may lead to decreased fertilization ability. Acrosomeless round-headed spermatozoa (globozoospermic spermatozoa) are unable to fertilize the oocytes, and increased percentages of morphologically abnormal acrosomes were related to fertilization failure in assisted reproductive technology (ART) using conventional in vitro fertilization (IVF) [36]. The AR is a time-dependent phenomenon that cannot take place prematurely or too late [37]. Premature acrosome reaction and the inability of the spermatozoa to release the acrosomal contents in response to proper stimuli (acrosome reaction insufficiency) have been associated with idiopathic male infertility [38]. Although the cause of premature acrosome reaction is unknown, the premature (stimulusindependent) initiation of acrosomal exocytosis seems to be related to a perturbation of the plasma membrane stability. In this situation, the acrosome reaction may not involve a premature activation of the receptor-mediated process, but rather reflect an inherent fragility of the sperm membrane, leading to a receptor-independent acrosomal loss [39]. Antisperm antibodies (ASA) may also adversely affect the ability of sperm to undergo capacitation and acrosome reaction [40]. Chang et al. [41] reported reduction in fertilization rate either by $\operatorname{IgG}$ directly bound to sperm or $\operatorname{IgM}$ present in female serum. The combination of $\operatorname{IgG}$ and IgA may have a synergistic negative effect on fertilization [42-45]. Toxic substances to sperm can also impact the AR. High concentrations of dietary phytochemicals, such as genistein isoflavone and $\beta$-lapachone, were shown to suppress the AR in a dose and time-dependent manner in the rat model [46]. Inhibition of AR by genistein seems to involve the protein kinase $\mathrm{C}$ pathway while $\beta$-lapachone has a direct cytotoxic effect on sperm cell membrane. It is suggested that genistein and $\beta$-lapachone may impact male fertility via AR suppression in high doses and AR induction in low doses [46]. Calcium channel blockers may also interfere with the AR exocytic event. Sperm incubation with different blockers, such as trifluoperazine (calmodulin inhibitor), verapramil $\left(\mathrm{Ca}^{2+}\right.$ channel inhibitor) and nifedipine (voltage-dependent $\mathrm{Ca}^{2+}$ channel inhibitor) significantly reduced the ability of hamster sperm to undergo the acrosome reaction [47].

\section{LABORATORY TESTS TO MEASURE HUMAN SPERM ACROSOME REACTION}

The assessment of the sperm acrosome reaction in vitro (AR) has been used as a test of sperm function for many years $[4,48]$. AR testing has been shown to be useful not only as a tool in andrology for research purposes, such as male contraception [49] and gonadotoxic effects of food and drugs [46] but also to predict fertilization success in assisted conception cycles. The AR testing is based on sperm physio- 
logy; it involves capacitation and acrosome reaction [50]. The sperm acrosome can be visualized by phase-contrast light microscopy. However, greater accuracy can be achieved by using either electron and fluorescence microscopy or monoclonal antibodies and simple dyes [50].

\section{Acrosome Reaction Testing Using Fluorescein-Labeled Lectins}

Fluorescein isothiocyanate-conjugated peanut agglutinin (FITC-PNA) or fluorescein isothiocyanate-conjugated pisum sativum (FITC-PSA) may be used to assess the sperm acrosome. FITC-PNA binds specifically to the outer acrosomal membrane (beta-fraction of D-galactose) while FITC-PSA binds to the alpha-metilmannose and labels the acrosome contents [8]. One of the most important aspects involving AR testing is the difficulty in differentiating between pathologic acrosomal loss, secondary to a reduction in cell viability, and a 'true' acrosome loss. We have demonstrated that a discrepancy exists between the proportion of acrosome-reacted spermatozoa in viable and overall sperm specimens [50] (Fig. 1). Dead spermatozoa lose its acrosomal contents by membrane rupture. Consequently, fluorochromes targeted to the acrosome membranes bind to receptors and the AR pattern is observed on fluorescence examination. The differentiation between 'pathological' and 'true acrosomal loss' is particularly important when assessing the acrosome status of cryo-thawed spermatozoa because cryopreservation directly damages sperm membrane resulting in the loss of membrane permeability and subsequent cell death [51]. It is,

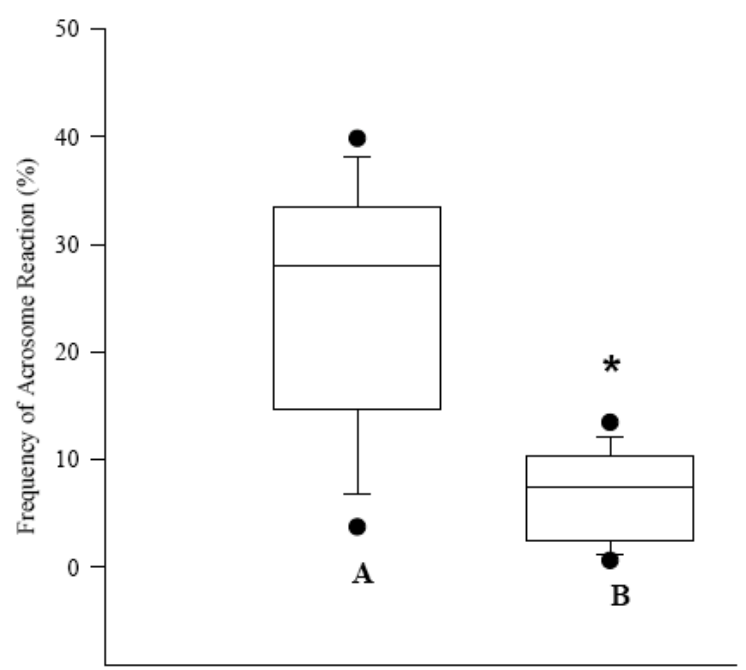

Fig. (1). The frequency of acrosome reaction (AR) in semen specimens of fertile donors. AR was determined by FITC-PNA, and sperm viability by Hoechst-33258 staining. (A) Total acrosome reaction (normal reacted cells plus post mortem degeneration of the acrosomes) and (B) acrosome reaction in live human spermatozoa (* $\mathrm{p}<0.01$ ). Box covers the middle $50 \%$ of the data values, between the lower and upper quartile. The central line is the median and the whiskers extend out to $80 \%$ of the data. Bars represent values between the 5 th and $95^{\text {th }}$ percentile (Adapted from International Braz J Urol, Vol. 33, 3, Esteves SC et al. Evaluation of acrosomal status and sperm viability in fresh and cryopreserved specimens by the use of fluorescent peanut agglutinin lectin in conjunction with hypo-osmotic swelling test, page 371, Copyright 2007, with permission from FB Sampaio, editor). therefore, recommended that supravital dyes are used in conjunction to fluorochromes to simultaneously monitor viability during AR assessment.

Cross et al. described the use of the supravital stain Hoechst-33258 (a fluorescent DNA-binding dye with limited membrane permeability) combined with immuno fluorescence technique for evaluating the acrosome reaction in viable spermatozoa [52]. A simple alternative to evaluate viability of acrosome reacted sperm cells is by using the hypo-osmotic swelling test (HOST) [53]. Sperm specimens are incubated in hyposmotic conditions and smears are prepared on to glass microscope slides. Sperm membranes are permeabilized in ice-cold methanol and then the fixed smears are immersed in a $40-\mu \mathrm{g} / \mathrm{mL}$ FITC-PNA solution. Each spermatozoon is first examined for tail swelling using phase contrast with halogen illumination. Then, illumination is changed to mercury ultraviolet epi-illumination source for assessing FITC-PNA staining. Spermatozoa are classified as osmotically competent if tail swelling is observed after exposure to the hyposmotic solution. The percentage of sperm exhibiting tail swelling after incubation under hyposmotic conditions are correlated with the percentage of spermatozoa with intact membranes, as it has been shown that HOST can also be used to assess sperm viability in fresh semen [54]. In a recent study, we determined the usefulness of the HOST as a marker of sperm viability for AR testing in fresh and frozen specimens [50]. HOST has potential advantages over

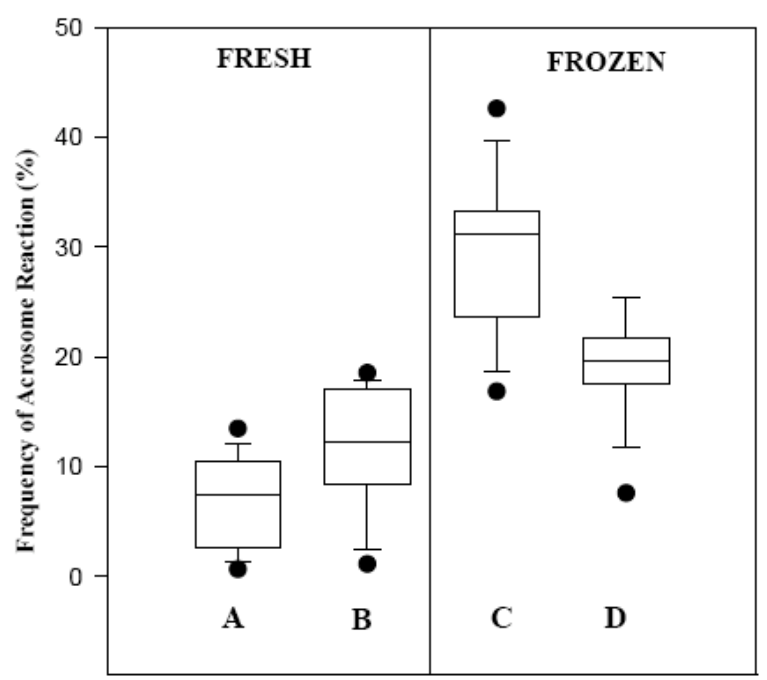

Fig. (2). Frequency of acrosome reaction in live human spermatozoa from 11 samples of fertile donors, determined by (A) FITC-PNA and HOST, and (B) FITC-PNA and Hoechst-33258, in fresh and post-thaw specimens. Similar acrosome reaction rates were detected by both $\mathrm{A}$ and $\mathrm{B}$ methods in fresh specimens $(\mathrm{P}=$ 0.07). In frozen specimens, the frequencies of acrosome reaction in viable spermatozoa assessed by A and B methods were significantly different $(\mathrm{p}=0.01)$. Box covers the middle $50 \%$ of the data values between the lower and upper quartile. The central line is the median and the whiskers extend out to $80 \%$ of the data. Bars represent values between the 5th and 95th percentile - (Adapted from International Braz J Urol, Vol. 33, 3, Esteves SC et al. Evaluation of acrosomal status and sperm viability in fresh and cryopreserved specimens by the use of fluorescent peanut agglutinin lectin in conjunction with hypo-osmotic swelling test, page 372, Copyright 2007, with permission from FB Sampaio, editor). 
protocols involving the use of fluorochrome-dye exclusion techniques. It precludes the use of an additional fluorescence filter cube to simultaneously assess the acrosome and to monitor viability. Our data showed that acrosome reaction determination was accurate by using FITC-PNA in conjunction to HOST to monitor viability in fresh sperm specimens, as validated by Hoescht-33258. However, AR results were not correlated in frozen-thawed spermatozoa (Fig. 2).

We have previously demonstrated the usefulness of the HOST to monitor sperm viability. Fresh and frozen-thawed sperm specimens were incubated with HOST and Hoescht33258 , and viability results were further validated by eosinnigosin dyes. The results of HOST and Hoescht-33258, and HOST and eosin-nigrosin, were highly correlated in unprocessed and processed fresh sperm specimens $(\mathrm{r}=0.95)$, but not in frozen-thawed ones ( $\mathrm{r}=0.22)$ [54] (Fig. 3).

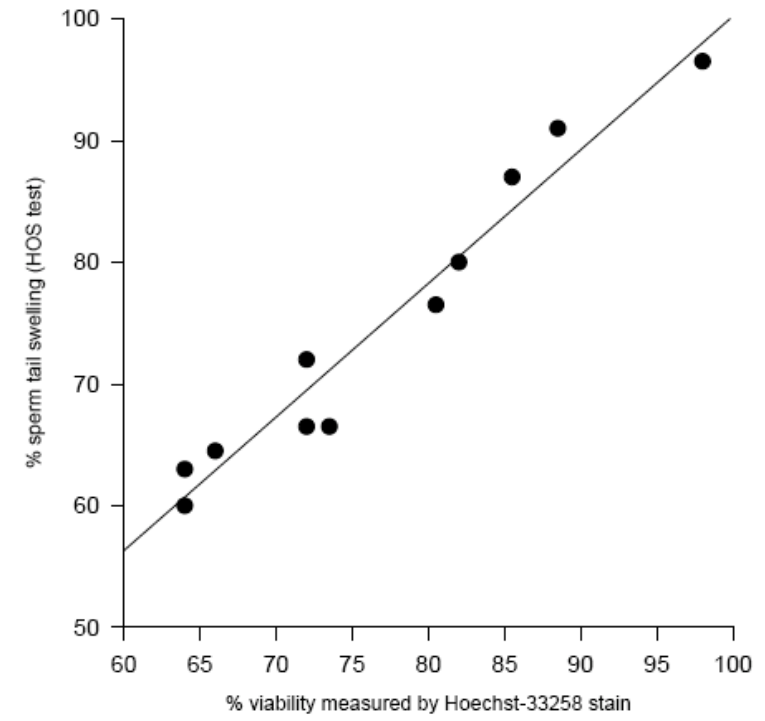

Fig. (3A). Correlation of viability results between sperm tail swelling by HOST and Hoechst 33258 in fresh semen specimens. Strong correlation seen between HOST and Hoechst $33258(\mathrm{r}=$ 0.95, $\mathrm{p}<0.001$ ). (Adapted from Fertil Steril, 1996, Vol. 66, Esteves $S C$, et al. Suitability of the hypo-osmotic swelling test to assess the viability of cryopreserved sperm, pp. 798-804, 1996).

The reasons for the poor HOST specificity for viability assessment in cryopreserved human sperm are unknown, but we speculate that sperm head and tail membranes have different liabilities to the freeze-thawing process. It may be possible that the determining factor for sperm survival after cryopreservation is the membrane integrity of the sperm head, which is not assessed by HOST. From these observations, we concluded that FITC-PNA labeling in conjunction with the HOST can accurately evaluate the acrosome reaction in viable fresh human spermatozoa, but not in cryopreserved ones. Protocols involving the use of supravital stains are recommended for simultaneous assessment of AR and to monitor viability in frozen-thawed spermatozoa [50]. In these circumstances, we prefer to use FITC-PNA associated to Hoechst-33258. Briefly, an aliquot of the sperm specimens are centrifuged and resuspended in $2 \mu \mathrm{g} / \mathrm{mL}$ Hoechst-33258 solution. The sperm suspension is incubated for 10 minutes in the dark and then spermatozoa are washed

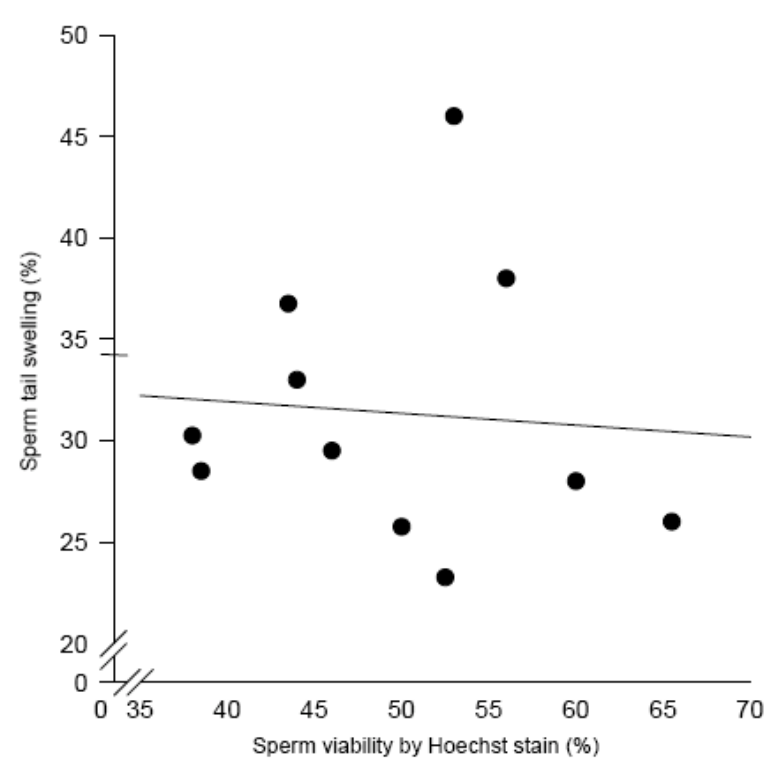

Fig. (3B). Correlation of viability results between sperm tail swelling by HOST and Hoechst 33258 stain in frozen-thawed semen specimens. No correlation was seen between HOST and Hoechst $33258(\mathrm{r}=0.11, \mathrm{p}=0.70)$. (Adapted from Fertil Steril, 1996, Vol. 66, Esteves SC, et al. Suitability of the hypo-osmotic swelling test to assess the viability of cryopreserved sperm, pp. 798804).

to remove excess stain. Smears are prepared on to glass microscope slides and then immersed in ice-cold methanol to permeabilize the sperm membranes. The fixed smears are immersed in a $40-\mu \mathrm{g} / \mathrm{mL}$ FITC-PNA solution, incubated at room temperature, and washed gently in PBS to remove the excess label [50]. A microscope equipped with phase contrast and fluorescence epi-illumination module is used to examine the slides at 1000X magnification. The illumination is selected to mercury ultraviolet epi-illumination source for assessing FITC-PNA and Hoechst-33258 labeling by using appropriate filter cubes. FITC-PNA and Hoechst-33258 fluoresce "apple-green" and bright medium blue, respectively (Fig. 4). Examination of the same spermatozoon for FITC-PNA labeling and for Hoechst-33258 staining is performed by interchanging the fluorescence filter cubes.

\section{Acrosome Reaction Challenge Testing}

Acrosome reaction can be induced in vitro by ionophores such as A23187 which exchange $\mathrm{Ca}^{2+}$ for $2 \mathrm{H}^{+}$. The ionophore-induced acrosome reaction, also termed 'acrosome reaction to ionophore challenge (ARIC)' has been extensively validated as a sperm function test with excellent predictive values for outcomes in assisted reproduction [4, 48, 55-58]. Briefly, motile spermatozoa are first capacitated for $3 \mathrm{~h}$ by incubation in sperm culture medium at $37^{\circ} \mathrm{C}$ and then divided into two equal aliquots: one is incubated with $2.5 \mu \mathrm{mol} / \mathrm{L}$ calcium ionophore A23187 solution, and the other is treated with dimethylsulfoxide $(10 \%, \mathrm{v} / \mathrm{v})$ as a control $[35,59,60]$. Acrosome status can be determined by FITC-PNA or FITC-PSA in combination with the nuclear stain bis-benzimide or HOST to monitor viability. The ARIC test score is calculated by subtracting the frequency of AR occurrence following ionophore challenge from the baseline values. Using the acrosome reaction to ionophore 


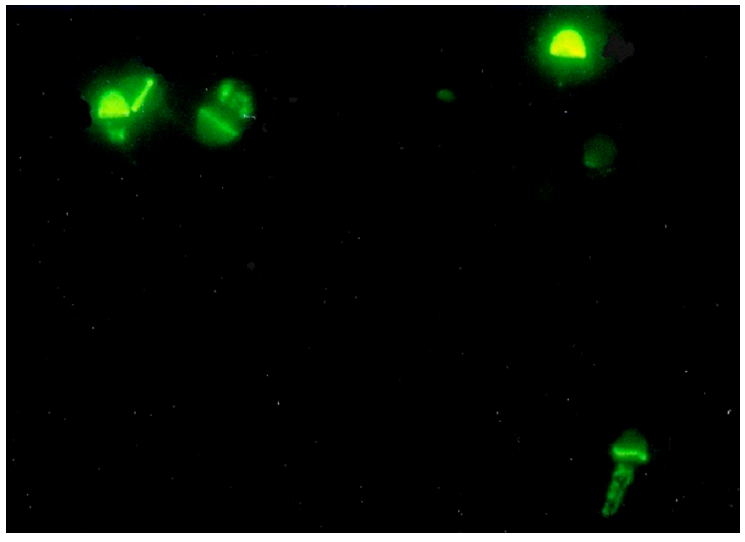

Fig. (4A). Photomicrograph of spermatozoa labeled by FITC-PNA. Spermatozoa were examined under fluorescence epi-illumination at $1,000 x$ magnification. In spermatozoa with intact acrosomes, the acrosomal region of the sperm head exhibited a uniform applegreen fluorescence. In spermatozoa exhibiting acrosome reaction, only the equatorial segment of the acrosome was stained (Adapted from International Braz J Urol, Vol. 33, 3, Esteves SC et al. Evaluation of acrosomal status and sperm viability in fresh and cryopreserved specimens by the use of fluorescent peanut agglutinin lectin in conjunction with hypo-osmotic swelling test, page 367, Copyright 2007, with permission from FB Sampaio, editor).

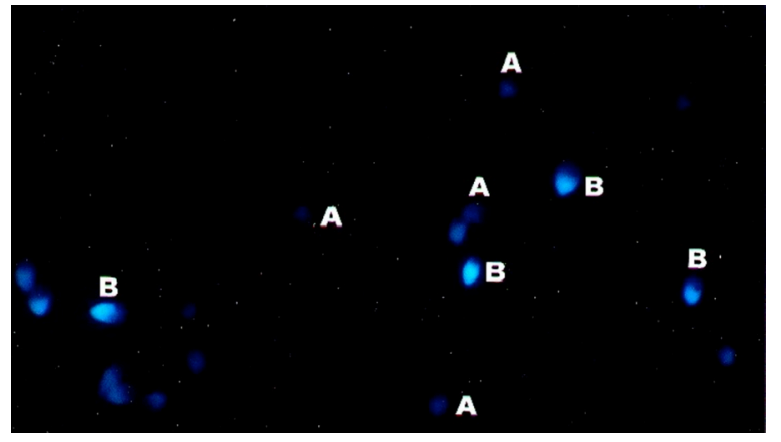

Fig. (4B). Photomicrograph of spermatozoa labeled by Hoechst33258. Spermatozoa were examined under mercury ultraviolet epiillumination at $1,000 \mathrm{x}$ magnification. Hoechst-33258 stains the nuclei of damaged cells (dead spermatozoa), which show a bright blue-white fluorescence (B) and is excluded from viable cells (live spermatozoa), which show a pale blue fluorescence (A). (Adapted from International Braz J Urol, Vol. 33, 3, Esteves SC et al. Evaluation of acrosomal status and sperm viability in fresh and cryopreserved specimens by the use of fluorescent peanut agglutinin lectin in conjunction with hypo-osmotic swelling test, page 367, Copyright 2007, with permission from FB Sampaio, editor).

challenge (ARIC) cut-off of $10 \%$, the sensitivity of the test in predicting subfertility is $54 \%$ with a positive predictive value of $64 \%$ and specificity of $85 \%$ [56]. Patients with ARIC scores above $10 \%$ have consistently better fertilization than those with lower scores $[4,48]$. The mechanism of the acrosome reaction induced by ionophores differs from the physiological acrosome reaction induced by the zona pellucida [18]. While the physiological acrosome reaction involves activation of several signal transduction pathways, the ionophore-induced acrosome reaction mainly involves a chemical effect on calcium influx that partially reflects the physiological acrosome reaction process [18].
Jaiswal et al. [61] compared different acrosome reaction inducers and probes to assess the acrosome reaction. They confirmed that different inducers potentiate different levels of acrosome reaction. Furthermore, their study provided an explanation for these differences by demonstrating that some inducers (e.g. progesterone and follicular fluid) trigger only partial acrosome reaction whereas calcium inophore A23187 induce both complete and partial acrosome reaction. This appears to be the reason why, in earlier studies, A23187 always yielded higher acrosome reaction levels than progesterone and follicular fluid [62]. It is therefore suggested that inducers such as progesterone and follicular fluid should be used for potentiating partial acrosome reaction, and the assessment should be carried out with probes that target the acrosomal content, e.g. FITC-PSA or FITC-PNA. For inducing a complete acrosome reaction, A23187 is preferable [61].

\section{IMPACT OF SPERM PROCESSING AND IN VITRO INCUBATION ON ACROSOME INTEGRITY}

In vivo, spermatozoa must be washed free from the seminal plasma before they are capable of undergoing capacitation and acrosome reaction. This process is accomplished under normal physiological conditions when seminal plasma is removed as the spermatozoa traverse the cervical mucus, and capacitation occurs as they are transported across the cervix, uterus and fallopian tubes [5]. Such conditions can be mimetized in vitro by sperm processing and incubation with protein-supplemented culture media. We sought to determine whether the swim-up technique selected spermatozoa with better motion characteristics, and how swim-up and subsequent capacitation affected the spontaneous acrosome reaction rate. The motile sperm fraction from normal donors was isolated by the swim-up method from a washed sperm pellet [59]. Then, specimens were capacitated for 3 hours by incubation in modified Biggers-Whitten-Whittingam (BWW) medium supplemented with 3\% human serum albumin at $37^{\circ} \mathrm{C}$ in a $5 \% \mathrm{CO} 2$ atmosphere. Sperm motility, viability, and motion characteristics, as assessed by computer-assisted semen analysis (CASA) were significantly increased in both swim-up and capacitated specimens. The percentage of acrosome-reacted spermatozoa remained unchanged $(\sim 13 \%)$ in both unprocessed and swim-up groups, but was significantly increased by $36 \%$ after capacitation. We concluded that swim-up isolates sperm with greater motion characteristics but does not change the acrosome reaction rate [63]. We also reported that in vitro capacitation is not an absolute prerequisite for an agonist-induced acrosome reaction using calcium ionophore, although capacitation may significantly optimize the agonist-induced acrosome reaction rate. Our data suggest that artificially-induced in vitro acrosome reaction may involve other complex mechanism(s) rather than a physiological change induced by capacitation, as also reported by others $[34,64]$.

\section{ACROSOME REACTION RESPONSE TO STIMU- LANTS}

Cyclic nucleotide phosphodiesterases (PDE) regulate intracellular levels of cAMP and cGMP by hydrolyzing them to the corresponding 5 ' monophosphates [65]. Nine different 
PDE isoenzymes ( $\mathrm{PDE}_{1}$ to $\left.\mathrm{PDE} 9\right)$ have been described and found to be present at various concentrations in human tissues $[66,67]$. Previous studies have shown that mRNA coding for cAMP-specific PDE $\left(\mathrm{PDE}_{4} \mathrm{~A}\right)$ isoforms are present in mature rat and mouse germ cells [68], and that the expression of these isoforms is maximal in round spermatids and is maintained in mature spermatozoa [69]. Nitric oxide synthase and PDE types 1, 4 and 5 isoforms are present in human spermatozoa [70, 71]. In vitro inhibition of sperm $\mathrm{PDE}_{1}, \mathrm{PDE}_{4}$ and $\mathrm{PDE}_{5}$ isoenzymes by specific inhibitors stimulates acrosome reaction and sperm motility [65, 71-73], suggesting that this is not a response specific to only one of the PDE family [74].

The mechanism by which PDE inhibitors exert their pharmacological effects is by inhibiting phosphodiesterase, an enzyme responsible for the degradation of cGMP and cAMP [75]. This creates an environment with increased energy substrates. Increased levels of cAMP and cGMP affect many intracellular functions including calcium transport [76]. This altered level of calcium is known to affect sperm motion and an energy-dependent influx of calcium into the sperm cell is believed to be responsible for initiation of the acrosome reaction $[30,77]$.

\section{cAMP Phosphodiesterase Inhibitors}

Cyclic AMP has a role in sperm kinematics and in the acrosome reaction second-messenger system $[30,78]$. Garbers et al. [79] made the initial observations that sperm motility could be increased by a variety of putative cyclic nucleotide phosphodiesterase inhibitors (caffeine, theophylline, papaverine). Treatments that increase intra-cellular cAMP concentrations often cause an increase in sperm motility and kinematics as well as in the agonist-induced acrosome reaction [30] and fertilization rates [80, 81]. Pentoxifylline (PF), a phosphodiesterase inhibitor, inhibits cyclic adenosine monophosphate (cAMP) phosphodiesterase, thus increasing intracellular cAMP concentration $[80,82]$ and tyrosine-phosphorylation at the tail level [83]. Also, it has been postulated that PF has the ability to scavenge reactive oxygen radicals $[60,84-86]$. Pentoxiffyline treatment increases the capacity of human spermatozoa to undergo to the acrosome reaction in response to both natural (follicular fluid) and artificial (ionophore A23187) stimuli, and it also improves the fertilizing ability in patients with acrosome reaction insufficiency [82, 87]. Significant increase in pregnancy rates were observed following intrauterine insemination when PF was added to the semen at the time of sperm preparation [88].

In humans, the ideal concentration of PF to be used in vitro is a matter of debate. Some authors reported beneficial effects with $3.6 \mathrm{mM}$ on motility and sperm fertilizing ability $[82,89,90]$ while others demonstrated a similar response both with lower and higher concentrations [91-94]. A detrimental effect on sperm membrane integrity was reported at the PF concentration of $10 \mathrm{mM}$ [86], but a considerable variable response to $\mathrm{PF}$ is also seen in both normozoospermic [92] and oligozoospermic semen specimens [80]. At a concentration of $5 \mathrm{mM}$ PF has oxygen-free radical scavenging capacities by reducing the superoxide release from human spermatozoa following phorbol myristate acetate [85]. Direct addition of PF to sperm specimens was shown to optimize fertilization rates in assisted conception involving conventional in vitro fertilization [80,95] According to our data, PF does not alter the frequency of spontaneous acrosome reaction at a $5 \mathrm{mM}$ concentration when added directly to the semen of normal and infertile men $[35,60]$.

\section{cGMP Phosphodiesterase Inhibitors}

Cyclic GMP is less extensively studied than cAMP on sperm function. It has been suggested that cGMP regulates lipid metabolism in other vertebrate species [79]. When pig spermatozoa are incubated in a minimal culture medium, added oviduct fluid can cause up to 50 -fold increase of cGMP [96]. In the human, dibutyl cGMP induces acrosomal loss in a pathway independent of calcium influx when spermatozoa were first preincubated under capacitating conditions [29]. The concentration of cyclic GMP in ejaculated human semen is $<16 \mathrm{pmol} / 10^{6}$ cells, while the corresponding cyclic AMP concentration is $100 \mathrm{pmol} / 10^{6}$ cells [32, 33]. Although the concentration of cGMP in ejaculated human sperm is almost 7 times lower than cAMP, it seems reasonable to speculate that both nucleotides may have a similar role, since cyclic AMP- and cGMP-dependent protein kinases, that mediate the actions of these nucleotides in various tissues, are similar in many respects and may be closely related proteins [34].

Sildenafil, a selective type 5 phosphodiesterase inhibitor (PDE5), acts by increasing intracellular levels of cGMP and cAMP [97]. Taken orally, it causes a marked increase in cGMP concentrations and facilitates erection in men with erectile dysfunction [98]. Acute administration of $100 \mathrm{mg}$ sildenafil promotes a concentration of $0.1-0.3 \mu \mathrm{mol} / \mathrm{L}$ in the ejaculate (Pfizer, Viagra ${ }^{\mathrm{TM}}$ data sheet), and this concentration is consistent with a possible inhibitory effect on sperm PDE isoforms [67]. The effects of sildenafil on human sperm function have gained attention in recent years. Cuadra et al. [72], studying its effects on sperm motility and acrosome parameters in vitro, observed a dose-dependent improvement on sperm motility and an increase in the spontaneous acrosome reaction rate. Aversa et al. [99] evaluated the effects of oral sildenafil administration $(100 \mathrm{mg})$ on seminal parameters in young healthy male volunteers. The authors found that sildenafil caused no changes in the seminal parameters when compared to placebo. Conversely, Lefievre et al. [100] reported that sildenafil stimulated human sperm motility and capacitation but not acrosome reaction at concentrations of $10,30,100$, or $200 \mu \mathrm{M}$. Recently, Glenn et al. [74] reported that sildenafil citrate improved sperm motility and increased the premature acrosome reaction rate in vitro at the concentration of 0.67 $\mu \mathrm{M}$.

We investigated the in vitro effects of sildenafil citrate on sperm function of healthy male volunteers with proven fertility. Semen specimens were obtained by masturbation and were divided into four equal aliquots: the first received no treatment (control) and the others were incubated with $0.001 \mu \mathrm{M}, 0.01 \mu \mathrm{M}$ and $0.1 \mu \mathrm{M}$ of sildenafil citrate (Pfizer, Sandwich, UK) for 1 hour. After treatment, all aliquots were processed by the swim-up technique and underwent further in vitro incubation under capacitating conditions for 3 hours. Overall, motion parameters assessed by CASA were significantly increased after sperm treatment with $0.01 \mu \mathrm{M}$ 
Table 1. Effects of Sperm Treatment with Sildenafil Citrate on Acrosome Integrity and Acrosome Reaction in Response to Ionophore Challenge (ARIC test) in 21 Normozoospermic Men (Adapted from Fertil Steril 84, Suppl, Esteves et al. Acrosome integrity and agonist-induced acrosome reaction rate after stimulation by varying concentrations of a specific inhibitor of cGMP phosphodiesterase type-5 (Sildenafil) in normozoospermic specimens, page S225, Copyright 2005, with permission from Elsevier)

\begin{tabular}{|c|c|c|c|c|c|c|}
\hline Sperm Parameters & Control & Sd1 [0.001 $\mu$ Molar $]$ & Sd2 [0.01 $\mu$ Molar $]$ & Sd3 [0.1 $\mu$ Molar $]$ & P-value* & P value \\
\hline $\begin{array}{l}\text { Frequency of live sperm with } \\
\text { intact acrosome (\%) }\end{array}$ & $82.0 \pm 8.9^{\mathrm{a}}$ & $88.8 \pm 6.8^{\mathrm{b}}$ & $84.8 \pm 7.5^{\mathrm{c}}$ & $88.5 \pm 7.8^{\mathrm{d}}$ & $\begin{array}{l}\mathrm{axb}=0.20 \\
\mathrm{axc}=0.15 \\
\mathrm{axd}=0.25\end{array}$ & 0.10 \\
\hline Acrosome reaction before ARIC test (\%) & $7.1 \pm 4.8^{\mathrm{a}}$ & $4.1 \pm 3.1^{\mathrm{b}}$ & $4.4 \pm 3.2^{\mathrm{c}}$ & $3.6 \pm 1.2^{\mathrm{d}}$ & $\begin{array}{l}\mathrm{axb}=0.02 \\
\mathrm{axc}=0.02 \\
\mathrm{axd}=0.01\end{array}$ & 0.26 \\
\hline Acrosome reaction after ARIC test (\%) & $24.8 \pm 14.7^{\mathrm{a}}$ & $30.6 \pm 20.8^{b}$ & $26.6 \pm 14.3^{\mathrm{c}}$ & $29.3 \pm 13.1^{\mathrm{d}}$ & $\begin{array}{l}\mathrm{axb}=0.75 \\
\mathrm{axc}=0.24 \\
\mathrm{axd}=0.60\end{array}$ & 0.52 \\
\hline Percent variation (\%) & $351.6 \pm 346.6^{\mathrm{a}}$ & $950.8 \pm 684.1^{\mathrm{b}}$ & $633.3 \pm 589.0^{\mathrm{c}}$ & $769.2 \pm 401.3^{\mathrm{d}}$ & $\begin{array}{l}\mathrm{axb}=0.03 \\
\mathrm{axc}=0.04 \\
\mathrm{axd}=0.04\end{array}$ & 0.09 \\
\hline
\end{tabular}

* Control versus Sildenafil (Sd) at different concentrations; a-d: pairwise comparisons by ANOVA

- Comparisons among different concentrations of sildenafil

$\mathrm{P}</=0.05$ was considered significant.

sildenafil. Curvilinear velocity, amplitude of lateral had displacement and hyperactivated motility were also significantly increased at the concentration of $0.1 \mu \mathrm{M}$, but no effects were observed at the sildenafil concentration of 0.001 $\mu \mathrm{M}$ [101]. Acrosome integrity and acrosome reaction in response to ionophore challenge (ARIC test) was examined by FITC-PNA combined with Hoechst-33258 to monitor sperm viability. Spontaneous acrosome reaction rates were similar in both control and treated groups. However, acrosome reaction rates in response to calcium-ionophore challenge were significantly higher in all sildenafil treated groups compared to control $(\mathrm{p}<0.05)$. A dose-dependent effect of type-5 PDE inhibitor on sperm acrosome status was not observed (Table 1) [102].

\section{SPERM CRYOPRESERVATION AND ACROSOME STATUS}

The freeze-thawing process directly damages the sperm membranes; as such, loss of membrane permeability and subsequent cell death may occur [51]. Cryopreservation can also cause permanent functional damage (sublethal damage), thus reducing the fertilizing ability of human sperm [35]. Cryopreservation-related impairment in sperm function can be explained partially by the reduction in the percentage of normal intact acrosomes [64]. Although acrosomal damage may be secondary to cell death [51] an increase in the proportion of viable spermatozoa with reacted acrosomes has been reported after cryopreservation [64]. Therefore, adequate assessment of the acrosomal status in cryopreserved sperm [50] should include monitoring of sperm viability, as previously discussed. Cryo-thawed spermatozoa may also have defective sperm function due to elevated levels of reactive oxygen specimens [103]. This may cause peroxidation of unsaturated fatty acids in the acrosomal membrane and decrease the response to calcium influx signals that trigger the acrosome reaction [35].

Efforts to improve the fertilizing ability of cryopreserved spermatozoa are useful for assisted conception. We have investigated different strategies to minimize the acrosomal loss due to the freeze-thawing process in human sperm. In one study, we examined the impact of freezing sperm after removing the seminal plasma and selecting the motile fraction on the post-thaw acrosome status [59]. It has been shown that prolonged exposure to seminal plasma results in marked decline in both motility and viability, and inhibition of the acrosome reaction [4, 48, 62, 104, 105]. Ejaculated spermatozoa are also damaged by reactive oxygen species emanating from seminal leukocytes and damaged spermatozoa [106]. In assisted reproduction procedures such as intrauterine insemination (IUI) and IVF, ejaculated spermatozoa are separated from the seminal environment as soon as possible. The swim-up procedure accomplishes this, and enables the selection of populations of highly motile spermatozoa for assisted reproduction procedures. To examine if the selection of such a sperm population before freezing minimized the deleterious effects of cryopreservation, we divided semen specimens from normal donors into two equal aliquots: one received no treatment (control) and the other was processed by swim-up from a washed sperm preparation [59]. Both aliquots were cryopreserved by the liquid nitrogen vapor method. Percentage motility and motion characteristics were evaluated by computer-assisted semen analysis. Acrosome integrity as well as spontaneous and calcium ionophore-induced acrosome reactions before freezing and after thawing were assessed by fluorescein isothiocyanate conjugated peanut agglutinin combined with a supra vital dye (Hoechst-33258). We noted that swim-up processing enabled selection of a sperm population with better motion characteristics, percentage motility and viability before freezing $(\mathrm{P}<0.001)$, but with no difference in percentage of acrosome-intact spermatozoa $(\mathrm{P}=0.63)$. After thawing, the 
Table 2. Effects of Pre-Freeze Sperm Processing by Swim-Up on Post-Thaw Calcium Ionophore induced Acrosome Reaction in 15 Normozoospermic Men (Adapted from Hum Reprod 15, 10, Esteves SC et al. Improvement in motion characteristics and acrosome status in cryopreserved human spermatozoa by swim-up processing before freezing, page 2177, Copyright 2000, with permission from Oxford University Press)

\begin{tabular}{|c|c|c|c|c|}
\hline Post-Thaw Frequency of Live Spermatozoa with Intact Acrosome (\%) & $\begin{array}{c}\text { Control group } \\
(\text { mean } \pm \text { SD })\end{array}$ & $\begin{array}{l}\text { Swim-up group } \\
\quad(\text { mean } \pm \text { SD })\end{array}$ & $\begin{array}{l}\text { Percentage change }{ }^{\mathrm{a}} \\
\quad(\text { mean } \pm \text { SD })\end{array}$ & P-value ${ }^{b}$ \\
\hline Before ionophore challenge & $66.5 \pm 9.2$ & $72.1 \pm 7.2$ & $10.1 \pm 17.5$ & 0.04 \\
\hline Percent change $^{\text {a }}$ & $-17.1 \pm 11.2$ & $-27.6 \pm 13.2$ & - & 0.003 \\
\hline$P$ value ${ }^{c}$ & $<0.001$ & $<0.001$ & - & - \\
\hline
\end{tabular}

${ }^{\mathrm{a}}$ Calculated as mean of individual changes.

${ }^{\mathrm{b}} \mathrm{P}<0.05$ was considered significant between control and swim-up groups. (Student's paired t-test).

${ }^{\mathrm{c}} \mathrm{P}<0.05$ was considered significant between the frequency of live spermatozoa with intact acrosome before and after ionophore challenge. $\mathrm{NS}=$ not significant

swim-up specimens exhibited faster velocity and progression than untreated specimens $(\mathrm{P}<0.001)$. They also had higher percentages of spermatozoa with intact acrosomes and spermatozoa able to undergo acrosome reaction in response to calcium ionophore (Table 2). This effect were not explained by higher initial values for acrosome integrity, because pre-freeze acrosome integrity was similar in both sperm preparations. We speculated that the highly motile sperm population selected by swim-up may not be affected by oxygen radicals emanating from damaged leukocytes and from other spermatozoa susceptible to or damaged by lipid peroxidation. In a study using transmission electron microscopy, it was demonstrated [107] that the deleterious effects of cryopreservation on sperm acrosomes were less evident if spermatozoa had been frozen without seminal plasma. We concluded that the swim-up method using a washed sperm preparation before freezing offered the possibility of selecting spermatozoa with better post-thaw motility, acrosome integrity, and ability to undergo AR. Because the yield of spermatozoa obtained by the swim-up procedure is low, oligozoospermic specimens are not suitable to be frozen by this treatment strategy.

In other studies, we investigated the effects of pentoxifylline (PF) added directly to the seminal plasma before freezing on acrosome status of normal and abnormal semen specimens $[35,60]$. In these trials, semen specimens were divided into two equal aliquots: one received no treatment (control) while the other was incubated with $5 \mathrm{mM} \mathrm{PF}$ (treated). Both aliquots were cryopreserved by the liquid nitrogen vapor method. Acrosome integrity and spontaneous and calcium ionophore-induced acrosome reactions were assessed with fluorescein isothiocyanate-conjugated peanut agglutinin combined with a supra-vital dye (Hoechst-33258). In normal semen, a PF-induced protective effect on acrosome loss during the freeze-thaw process was observed (Table 3) [35].

In oligoasthenozoospermic specimens, however, a significant decrease in the post-thaw percentage of viable spermatozoa with intact acrosomes in both control and PFtreated specimens was observed [60]. In both normal and abnormal specimens, prefreeze sperm treatment with PF improved the ability of cryo-thawed spermatozoato undergo the acrosome reaction in response to stimuli (Tables 4 and 5). Interestingly, the ability of cryo-thawed sperm from abnormal specimens to respond to ionophore challenge was lower than that of normal sperm, irrespectively of treatment with PF before freezing. In normal semen, post-thaw acrosome reaction stimulated by calcium ionophore was $34.8 \%$ and $53.3 \%$ for control and treated specimens, respectively, while values of $11.8 \%$ and $30.2 \%$ were reported in abnormal semen specimens. In infertile men, ARIC scores were higher than $10 \%$ in $50 \%$ of the cryo-preserved PF-treated specimens, and in only $19 \%$ of the controls. In normal men, ARIC scores higher than $10 \%$ were seen in $80 \%$ and $60 \%$ of the cryo-thawed PF-treated and control specimens, respectively. PF improved ARIC scores in $81.2 \%$ and $100 \%$ of infertile and normal men, respectively. Our results suggest that normal semen resist cryo-injury better than specimens from oligoasthenozoospermic men. They also indicate that treatment of human sperm with PF may enhance post-thaw sperm fertilizing ability. PF at a 5-mM concentration afford greater cryo-protection in normal semen while a different

Table 3. Effects of Pre-Freeze Sperm Treatment with Pentoxifylline on Spontaneous Acrosome Reaction in 15 Normozoospermic Men before and after Cryopreservation (Adapted from Hum Reprod 13, 12, Esteves SC et al. Cryopreservation of human spermatozoa with pentoxifylline improves the post-thaw agonist-induced acrosome reaction rate, page 3386, Copyright 1998, with permission from Oxford University Press)

\begin{tabular}{|c|c|c|c|}
\hline Frequency of Spontaneous Acrosome Reaction & Control Median (IQ Range) & Pentoxifylline Median (IQ Range) & P value* \\
\hline \hline Pre-freeze & $10.3(4.5-15.5)$ & $17.8(14.7-25.5)$ & 0.0001 \\
\hline Post-thaw & $28.2(26.6-41.7)$ & $32.3(27.5-53.6)$ & 0.04 \\
\hline Percentage change & $219.5(163.9-495.2)^{* *}$ & $127.2(23.8-212.2)^{* *}$ \\
\hline
\end{tabular}

$*<0.05$ was considered significant (Wilcoxon signed-rank test).

** Significant increase after cryo-preservation $(\mathrm{P}<0.001)$.

IQ = interquartile. 
Table 4. Effects of Pre-Freeze Sperm Treatment with Pentoxifylline on Post-Thaw Calcium-Ionophore induced Acrosome Reaction in 15 Normozoosperrnic Men after Cryopreservation (Adapted from Hum Reprod 13, 12, Esteves SC et al. Cryopreservation of human spermatozoa with pentoxifylline improves the post-thaw agonist-induced acrosome reaction rate, page 3386 , Copyright 1998, with permission from Oxford University Press)

\begin{tabular}{|c|c|c|c|}
\hline Before ionophore challenge & $28.2(26.6-41.7)$ & $32.3(27.5-53.6)$ & 0.04 \\
\hline Percentage change & $34.8(10.3-57.8)^{* *}$ & $53.3(22.6-101.7)^{* *}$ & 0.03 \\
\hline
\end{tabular}

$*<0.05$ was considered significant (Wilcoxon signed-rank test).

** Significant increase after cryopreservation $(\mathrm{P}<0.001)$.

$\mathrm{IQ}=$ interquartile

Table 5. Effects of Pre-Freeze Treatment with Pentoxifyiine on Post-Thaw Calcium Ionophore-Induced Acrosome Reaction in Sperm from 16 Oligoasthenozoospermic Men (Adapted from Braz J Med Biol Res 2007; 40, 7, Esteves SC et al. Effects of pentoxifylline treatment before freezing on motility, viability and acrosome status of poor quality human spermatozoa cryopreserved by the liquid nitrogen vapor method, page 989, Copyright 2007, with permission from Creative Commons Attribution License)

\begin{tabular}{|c|c|c|}
\hline Post-Thaw Frequency of Live Spermatozoa with Reacted Acrosomes (\%) & Control & Pentoxifylline \\
\hline \hline Before ionophore challenge & $43.9(27.2-54.5)$ & $38.0(30.1-51.2)$ \\
\hline After ionophore challenge & $45.6(25.7-59.1)$ & $52.7(38.5-67.4)^{*}$ \\
\hline Percent change $^{\text {a }}$ & $-11.8(-(32.0-2.9))$ & $-30.2(-(40.8-18.5))^{*}$ \\
\hline ARIC score (\%) $^{*}$ & $4.8(0.5-6.8)$ & $9.7(6.6-19.7)^{*}$ \\
\hline P value $^{\text {b }}$ & $<0.001$ & $<0.001$ \\
\hline
\end{tabular}

of acrosome reaction occurrence after ionophore challenge from the frequency of occurrence before it.

a percent change calculated as median of individual changes;

${ }^{\mathrm{b}} \mathrm{P}$ value comparing frequency of live spermatozoa with reacted acrosomes before and after ionophore challenge (Wilcoxon signed-rank test).

$* \mathrm{P}<0.05$ compared to control (Wilcoxon signed-rank test).

concentration may be necessary to neutralize excessive reactive oxygenspecies (ROS) emanating from the already compromised spermatozoa from oligoasthenozoospermic individuals.

\section{EXPERT COMMENTARY}

The fertilization process is a net result of a series of molecular events enabling sperm to reach, recognize, bind to, and penetrate the oocyte. There has been a dramatic increase in our knowledge of the protein composition of the spermatozoon, its structures and the surrounding fluids (epididymal, vesicular, prostate) contributing to its function. As a result of research involving sperm capacitation and AR, we gained insight into the signalling complexes involved in key sperm physiological processes. Determination of both basal and induced-acrosome reaction may be clinically useful. A high proportion of sperm exhibiting either AR prematurity or stimuli-induced AR deficiency is correlated with decreased the sperm's fertilizing potential in vivo and in vitro. In assisted conception treatment cycles involving intrauterine insemination and conventional in vitro fertilization, the incubation interval after sperm processing should not exceed 3 hours. Longer post-processing incubation increases the proportion of sperm that undergo untimely acrosome reaction and may negatively impact treatment results.
Although specialized semen tests such as the AR aid in determining specific defects of human sperm physiology, its clinical use is limited nowadays by the success of ICSI for men with male infertility. As such, there is little incentive for infertility clinics to invest in elaborate sperm function tests. Although this policy is prevailing in the present time, ICSI is very expensive, invasive, has limited success, a number of side effects, is not widely available, and we still have little knowledge as to the effects of the use of ICSI on the health of future generations. The increasing demand to treat couples with the least invasive treatment, as posed by some government-funded infertility treatment policies, could encourage a step-back towards the use of prognostic sperm tests for both diagnosis and treatment. Currently, AR testing is rarely used in the clinical setting for men being evaluated after IUI or IVF failure to better discriminate those who may benefit from ICSI. The present focus of the in vitro AR testing is andrology research involving male contraception and gonadotoxic effects of food and drugs.

\section{FIVE-YEAR REVIEW}

Most studies involving the sperm acrosome reaction were published 20-30 years ago. This boom in research was compelled by the need to understanding the physiology of human fertilization in vivo and in vitro, as a consequence of the increasing demand of male infertility as an important 
health issue. Conventional semen analysis results, despite being an integral step of the male infertility evaluation, do not necessarily correlate with fertility potential because it does not assess sperm function. There are still a significant proportion of patients with normal and abnormal semen analyses showing unexplained infertility and achieving unassisted conception, respectively [108, 109]. In fact, it is not so much the absolute numbers of spermatozoa that determines fertility, but their functional competence. The acrosome reaction testing has been shown to be a stable parameter of sperm function and a valid tool to predict the fertilizing potential of human spermatozoa in vitro. In a recent study, calcium ionophore-induced acrosome reaction (ARIC) results have been shown to be a reliable indicator of fertility potential in intrauterine insemination and conventional IVF treatment cycles [110]. The AR threshold values determined using ROC curves for predicting complete fertilization failure and pregnancy in IVF were $21 \%$ and $26 \%$ respectively, and they were the sole predictors independent of the conventional semen analysis. In addition, no pregnancies were obtained by IUI when ARIC results were below $11 \%$. With ICSI, zona pellucida penetration and oolemmal fusion are bypassed and the role of acrosome reaction is unclear. ARIC results were not predictive of ICSI outcome, and sperm selection from different acrosomereacted sperm populations appeared to have no impact on fertilization rates $[15,110]$.

The AR assessment has been also used as an important biomarker in studies involving drugs and toxins. In one report, the frequency of acrosome-reacted spermatozoa was increased by sperm incubation with sildenafil citrate while in another the recreational use of delta-9-tetrahydrocannabinol caused partial inhibition of the sperm ability to undergo stimuli-induced AR [74, 111]. Falzone et al. [112], studying the effect of pulsed $900-\mathrm{MHz}$ GSM mobile phone radiation on the AR of human sperm, observed that although radiofrequency electromagnetic fields (RF-EMF) exposure did not adversely affect the AR rate, it had a significant effect on sperm morphometry and in the ability of sperm binding to the hemizona. Mukhopadhyay et al. (2010) evaluated the in vitro effect of benzo[a]pyrene, a substance present in cigarettes, on sperm hyperactivation and acrosome status of normozoospermic semen specimens. They observed a significant increase in sperm hyperactivation as well as in the premature acrosome reaction rates in presence of benzo[a]pyrene [113].

Recent publications on acrosome reaction focused on the biochemical and functional aspects of sperm-oocyte fusion. It has been demonstrated that in humans, ZP1 in addition to ZP3 and ZP4 binds to capacitated spermatozoa and induces acrosomal exocytosis [20, 21, 114, 115]. The ZP3-induced acrosome reaction involves the activation of T-type voltageoperated calcium channels (VOCCs), whereas ZP1- and ZP4-induced ARs involve both T- and L-type VOCCs. Chiu et al. [116] reported that glycodelin-A, a glycoprotein present in the female reproductive tract, sensitizes spermatozoa to the zona pellucida-induced acrosome reaction in a glycosylation-specific mechanism involving the activation of the adenylyl cyclase/PKA pathway, suppression of extracellular signal-regulated kinase activation and up-regulation of zona pellucida-induced calcium influx. It is therefore suggested that glycodelin-A may be important in vivo to ensure full responsiveness of human spermatozoa to the zona pellucida.

\section{KEY ISSUES}

- Capacitation prepares the sperm to undergo the acrosome reaction with the accompanying release of lytic enzymes and exposure of membrane receptors, which are required for sperm penetration through the zona pellucida and for fusion with the oolema. AR is a time-coordinated phenomenon that cannot occur prematurely or too late.

- When the process of AR is impaired, either by lack of an acrosome or acrosomal dysfunction, fertility can be compromised. Acrosome-reacted sperm lack the ability to bind to the ZP so are essentially infertile sperm.

- In vitro, the most widely used methods to assess the sperm acrosome utilize optical microscopy and fluorescent lectins staining that bind to either the outer or inner acrosomal membrane.

- Because acrosomal loss can be a result of sperm death, acrosome testing has to be used in conjunction with viability assays, such as supravital staining techniques and the hyposmotic swelling test, to distinguish non-viable sperm from 'truly' reacted acrosomes.

- HOST is a simple and reliable assay to monitor viability during AR assessment in fresh but not in cryopreserved semen.

- In IUI and conventional IVF, caution should be applied to avoid long post-processing incubation intervals before insemination. The proportion of sperm that undergo spontaneous AR acrosome reaction is increased after 3 hours of incubation.

- Several substances may affect the sperm acrosome, including cyclic AMP and GMP phosphodiesterase inhibitors, calcium ionophone and toxins.

- Strategies to minimize acrosomal loss due to the freeze-thawing process include the freezing of selected sperm populations and the addition of motility stimulants such as pentoxifylline.

- The assessment of the acrosome reaction in vitro may predict the sperm's fertilizing potential and is a potential marker to aid in the selection of assisted conception treatment. Currently, with the success of ICSI for male infertility AR testing is rarely used in the clinical setting. The increasing demand to treat couples with the least invasive treatment could encourage a step-back towards the use of such prognostic sperm tests for both diagnosis and treatment.

- In vitro AR testing is routinely used in andrology research involving male contraception and gonadotoxic effects of food and drugs.

\section{ACKNOWLEDGEMENTS}

None Declared. 


\section{CONFLICT OF INTEREST}

None Declared.

\section{REFERENCES}

[1] Toshimori K, Ito C. Formation and organization of the mammalian sperm head. Arch Histol Cytol 2003; 66(5): 383-96.

[2] WHO Laboratory manual for the examination of human semen and semen-cervical mucus interaction, $4^{\text {th }}$ ed. Cambridge: Cambridge University Press 1999; p. 19.

[3] Florman HM, Jungnickel MK, Sutton KA. Regulating the acrosome reaction. Int J Dev Biol 2008; 52(5-6): 503-10.

[4] Henkel R, Muller C, Miska W, Gips H, Schill WB. Determination of the acrosome reaction in human spermatozoa is predictive of fertilization in vitro. Hum Reprod 1993; 8(12): 2128-32.

[5] Mortimer D. From the semen to oocyte: the long route in vivo and in vitro short cut. In: Testart J, Frydman R, Eds. Human In Vitro Fertilization: Actual Problems and Prospects. Amsterdam: Elsevier Science Publishers 1985; p. 93.

[6] Fraser LR. Mechanisms controlling mammalian fertilization. In: Clarke JR, Eds. Oxford Reviews of Reproductive Biology. Oxford: Oxford University Press 1984; p. 173.

[7] Cross NL. Effect of cholesterol and other sterols on human sperm acrosomal responsiveness. Mol Reprod Dev 1996; 45(2): 212-7.

[8] Mortimer D. Practical laboratory andrology. New York: Oxford University Press 1994.

[9] Lamirande E, Leclerc P, Gagnon C. Capacitation as a regulatory event that primes spermatozoa for the acrosome reaction and fertilization. Mol Hum Reprod 1997; 3(3): 175-94.

[10] Lindemann, CB, Kanous, KS. Regulation of mammalian sperm motility. Arch Androl 1989; 23(1): 1-22.

[11] Matsumura K, Aketa K. Proteasome (multicatalytic proteinase) of sea urchin sperm and its possible participation in the acrosome reaction. Mol Reprod Dev 1991; 29(2): 189-99.

[12] Inaba K, Akazome Y, Morisawa M. Two high molecular mass proteases from sea urchin sperm. Biochem Biophys Res Commun 1992; 182(2): 667-74.

[13] Morales P, Kong M, Pizarro E, Pasten C. Participation of the sperm proteasome in human fertilization. Hum Reprod 2003; 18(5): 10107.

[14] Zaneveld LJ, De Jonge CJ, Anderson RA, Mack SR. Human sperm capacitation and the acrosome reaction. Hum Reprod 1991; 6(9): 1265-74.

[15] Mansour RT, Serour MG, Abbas AM, et al. The impact of spermatozoa preincubation time and spontaneous acrosome reaction in intracytoplasmic sperm injection: a controlled randomized study. Fertil Steril 2008; 90(3): 584-91.

[16] Yanagimachi R, Bhattacharyya A. Acrosome-reacted guinea pig spermatozoa become fusion competent in the presence of extracellular potassium ions. J Exp Zool 1988; 248(3): 354-60.

[17] Katz DF, Drobnis EA, Overstreet JW. Factors regulating mammalian sperm migration through the female reproductive tract and oocyte vestments. Gamete Res 1989; 22: 443.

[18] Breitbart H, Spungin B. The biochemistry of the acrosome reaction. Mol Hum Reprod 1997; 3(3): 195-202.

[19] Kopf GS, Gerton GL. The mammalian sperm acrosome and the acrosome reaction. In: Wasserman PM, Ed. Elements of mammalian fertilization. Boston: CRC Press 1991; pp. 153-203.

[20] Chiu PCN, Wong BST, Chung MK, et al. Effects of native human zona pellucida glycoproteins 3 and 4 on acrosome reaction and zona pellucida binding of human spermatozoa. Biol Reprod 2008; 79(5): 869-77.

[21] Ganguly A, Bukovsky A, Sharma RK, Bansal P, Bhandari B, Gupta SK. In humans, zona pellucida glycoprotein-1 binds to spermatozoa and induces acrosomal exocytosis. Hum Reprod 2010; 25(7): 164356.

[22] Yanagimachi, R. Mammalian fertilization. In: Knobil E, Neill JD, Ewing LL, et al., Eds. The physiology of reproduction. New York: Raven Press 1994; pp. 189.

[23] Brucker C, Lipford GB. The human sperm acrosome reaction: physiology and regulatory mechanisms. An update. Hum Reprod Update 1995; 1(1): 51-62.

[24] Thomas P, Meizel S. Phosphatidylinositol 4,5-bisphosphate hydrolysis in human sperm stimulated with follicular fluid or progesterone is dependent upon $\mathrm{Ca}^{2+}$ influx. Biochem J 1989; 264(2): 539-46.

Burrello N, Vicari E, D'Amico L, Satta A, D'Agata R, Calogero AE. Human follicular fluid stimulates the sperm acrosome reaction by interacting with the gamma-aminobutyric acid receptors [abstract]. Fertil Steril 2004; 82(Suppl 3): 1086-90.

[26] Aquila S, Sisci D, Gentile M, et al. Towards a physiological role for cytochrome P450 aromatase in ejaculated human sperm. Hum Reprod 2003; 18(8): 1650-9.

[27] Adeoya-Osiguwa SA, Markoulaki S, Pocock V, Milligan SR, Fraser LR. 17b-Estradiol and environmental estrogens significantly affect mammalian sperm function. Hum Reprod 2003; 18(1): 100-7.

[28] Baldi E, Luconi M, Bonaccorsi L, Muratori M, Forti G. Intracellular events and signaling pathways involved in sperm acquisition of fertilizing capacity and acrosome reaction. Front Biosci 2000; 1(5): 110-23.

[29] Bielfeld P, Anderson RA, Mack SR, De Jonge CJ, Zaneveld LJ. Are capacitation or calcium ion influx required for the human sperm acrosome reaction? Fertil Steril 1994; 62(6): 1255-61.

[30] De Jonge CJ, Han HL, Lawrie H, Mack SR, Zaneveld LJ. Modulation of the human sperm acrosome reaction by effectors of the adenylate cyclase/cyclic AMP second-messenger pathway. J Exp Zool 1991; 258(1): 113-25.

[31] Doherty CM, Tarchala SM, Radwanska E, De Jonge CJ. Characterization of two second messenger pathways and their interactions in eliciting the human sperm acrosome reaction. J Androl 1995; 16(1): 36-46.

[32] Robison GA, Sutherland EW. Cyclic AMP and the function of eukaryotic cells: an introduction. Ann N Y Acad Sci 1971; 185: 5-

[33] Gray JP. Cyclic AMP and cyclic GMP in gametes. Ph.D. Dissertation. Nashville (TN): Vanderbilt University 1971.

[34] Birnabaumer L, Pohl SL, Rodbell M. Adenyl cyclase in fat cells. Properties and the effects of adrenocorticotropin and fluoride. J Bioch Chem 1969; 244(13): 3468-76.

[35] Esteves SC, Sharma RK, Thomas AJ Jr, Agarwal A Cryopreservation of human spermatozoa with pentoxifylline improves the post-thaw agonist-induced acrosome reaction rate Hum Reprod 1998; 13(12): 3384-9.

[36] Kilani Z, Ismail R, Ghunaim S, et al. Evaluation and treatment of familial globozoospermia in five brothers. Fertil Steril 2004; 82(5): 1436-9.

[37] Cummins JM, Fleming AD, Crozet N, Kuehl TJ, Kosower NS, Yanagimachi R. Labelling of living mammalian spermatozoa with the fluorescent thiol alkylating agent, monobromobimane (MB): immobilization upon exposure to ultraviolet light and analysis of acrosomal status. J Exp Zool 1986; 237(3): 375-82.

[38] Tesarik J, Mendoza C. Alleviation of acrosome reaction prematurity by sperm treatment with egg yolk. Fertil Steril 1995; 63(1): 153-7.

[39] Esteves SC, Sharma RK, Thomas AJ Jr, Agarwal A. Effect of in vitro incubation on spontaneous acrosome reaction in fresh and cryopreserved human spermatozoa. Int J Fertil Womens Med 1998; 43(5): 235-42.

[40] Chiu WW, Chamley LW. Clinical associations and mechanisms of action of antisperm antibodies. Fertil Steril 2004; 82(2): 529-35.

[41] Chang TH, Jih MH, Wu TC. Relationship of sperm antibodies in women and men to human in vitro fertilization, cleavage, and pregnancy rate. Am J Reprod Immunol 1993; 30(2-3): 108-12.

[42] Junk SM, Matson PL, Yovich JM, Bootsma B, Yovich JL. The fertilization of human oocytes by spermatozoa from men with antispermatozoal antibodies in semen. J In Vitro Fert Embryo Transf 1986; 3(6): 350-2.

[43] Meinertz H, Linnet L, Fogh-Andersen P, Hjort T. Antisperm antibodies and fertility after vasovasostomy: a follow-up study of 216 men. Fertil Steril 1990; 54(2): 315-21.

[44] De Almeida M, Gazagne I, Jeulin C, et al. In-vitro processing of sperm with autoantibodies and in-vitro fertilization results. Hum Reprod 1989; 4(1): 49-53.

[45] Matson PL, Junk SM, Spittle JW, Yovich JL. Effect of antispermatozoal antibodies in seminal plasma upon spermatozoal function. Int J Androl 1988; 11(2): 101-6.

[46] Kumi-Diaka J, Townsend J. Toxic potential of dietary genistein isoflavone and beta-lapachone on capacitation and acrosome reaction of epididymal spermatozoa. J Med Food 2003; 6(3): 201-8. 
[47] Feng HL, Han YB, Hershlag A, Zheng LJ. Impact of $\mathrm{Ca}^{2+}$ flux inhibitors on acrosome reaction of hamster spermatozoa. J Androl 2007; 28(4): 561-4.

[48] Calvo L, Dennison-Lagos L, Banks SM, et al. Acrosome reaction inducibility predicts fertilization success at in-vitro fertilization. Hum Reprod 1994; 9(10): 1880-6.

[49] Suri A. Contraceptive vaccines targeting sperm. Expert Opin Biol Ther 2005; 5(3): 381-92.

[50] Esteves SC, Sharma RK, Thomas AJ Jr, Agarwal A. Evaluation of acrosomal status and sperm viability in fresh and cryopreserved specimens by the use of fluorescent peanut agglutinin lectin in conjunction with hypo-osmotic swelling test. Int Braz J Urol 2007; 33(3): 364-74.

[51] Cross NL, Hanks SE. Effects of cryopreservation on human sperm acrosomes. Hum Reprod 1991; 6(9): 1279-83.

[52] Cross NL, Morales P, Overstreet JW, Hanson FW. Two simple methods for detecting acrosome-reacted human sperm. Gam Res 1986; $15: 213-26$.

[53] Aitken RJ, Buckingham DW, Fang HG. Analysis of the responses of human spermatozoa to A23187 employing a novel technique for assessing the acrosome reaction. J Androl 1993; 14(2): 132-41.

[54] Esteves SC, Sharma RK, Thomas AJ Jr, Agarwal A. Suitability of the hypo-osmotic swelling test for assessing the viability of cryopreserved sperm. Fertil Steril 1996; 66(5): 798-804.

[55] Yovich JM, Edirisinghe WR, Yovich JL. Use of the acrosome reaction to ionophore challenge test in managing patients in an assisted reproduction program: a prospective, double-blind, randomized controlled study. Fertil Steril 1994; 61(5): 902-10.

[56] Cummins JM, Pember SM, Jequier AM, Yovich JL, Hartmann PE. A test of the human sperm acrosome reaction following ionophore challenge. Relationship to fertility and other seminal parameters. J Androl 1991; 12(2): 98-103.

[57] Liu DY, Baker HW. Defective sperm-zona pellucida interaction: a major cause of failure of fertilization in clinical in-vitro fertilization. Hum Reprod 2000; 15(3): 702-8.

[58] Katsuki T, Hara T, Ueda K, Tanaka J, Ohama K. Prediction of outcomes of assisted reproduction treatment using the calcium ionophore-induced acrosome reaction. Hum Reprod 2005; 20(2): 469-75.

[59] Esteves SC, Sharma RK, Thomas Jr AJ, Agarwal A. Improvement in motion characteristics and acrosome status in cryopreserved human spermatozoa by swim-up processing before freezing. Hum Reprod 2000; 15(10): 2173-9.

[60] Esteves SC, Spaine DM, Cedenho AP. Effects of pentoxifylline treatment before freezing on motility, viability and acrosome status of poor quality human spermatozoa cryopreserved by the liquid nitrogen vapor method. Braz J Med Biol Res 2007; 40(7): 985-92.

[61] Jaiswal BS, Eisenbach M, Tur-Kaspa I. Detection of partial and complete acrosome reaction in human spermatozoa: which inducers and probes to use? (1999). Mol Hum Reprod 1999; 5(3): 214-9.

[62] Parinaud J, Vieitez G, Moutaffian H, Richoilley G, Labal B. Variations in spontaneous and induced acrosome reaction. Correlations with semen parameters and in-vitro fertilization results. Hum Reprod 1995; 10(8): 2085-9.

[63] Esteves SC, Sharma RK, Thomas AJ Jr, Agarwal A. Effect of swim-up sperm washing and subsequent capacitation on acrosome status and functional membrane integrity of normal sperm. Int $\mathrm{J}$ Fertil Womens Med 2000; 45(5): 335-41.

[64] Chang MC. The meaning of sperm capacitation. A historical perspective. J Androl 1984; 5(2): 45-50.

[65] Conti M, Nemoz G, Sette C, Vicini E. Recent progress in understanding the hormonal-regulation of phosphodiesterases. Endo Rev 1995; 16(3): 370-89.

[66] Soderling SH, Bayuga SL, Beavo JA. Cloning and characterization of cAMP-specific cyclic nucleotide phosphodiesterase. Proc Natl Acad Sci USA 1998; 95(15): 8991- 6.

[67] Fabbri A, Aversa A, Isidori A. Sildenafil and erectile dysfunction. J Endocrinol Invest 1999; 22(6): 486-91.

[68] Naro F, Zhang R, Conti M. Developmental regulation of unique adenosine 3',5'-monophosphate-specific phosphodiesterase variants during rat spermatogenesis. Endocrinology 1996; 137(6): 2464-72.

[69] Soderling SH, Bayuga SL, Beavo JA. Identification and characterization of a novel family of cyclic nucleotide phosphodiesterase. J Biol Chem 1998; 273(25): 15553-8.

[70] Lewis SE, Donnelly ET, Sterling ES, Kennedy MS, Thompson W, Chakravarthy U. Nitric oxide synthase and nitrite production in human spermatozoa: evidence that endogenous nitric oxide is beneficial to sperm motility. Mol Hum Reprod 1996; 2(11): 873-8.

[71] Fisch JD, Behr B, Conti M. Enhancement of motility and acrosome reaction in human spermatozoa: differential activation by typespecific phosphodiesterase inhibitors. Hum Reprod 1998; 13(5): $1248-54$.

[72] Cuadra DL, Chan PJ, Patton WC, Stewart SC, King A. Type 5 phosphodiesterase regulation of human sperm motility. Am J Obstet Gynecol 2000; 182(5): 1013-5.

[73] Beavo JA. Cyclic-nucleotide phosphodiesterases: functional implications of multiple isoforms. Phys Rev 1995; 75(4): 725- 48.

[74] Glenn DRJ, McVicar CM, McClure N, Lewis SEM. Sildenafil citrate improves sperm motility but causes a premature acrosome reaction in vitro. Fertil Steril 2007, 87(5): 1064-70.

[75] Omote M. Pharmacological profiles of sildenafil (VIAGRA) in the treatment of erectile dysfunction: efficacy and drug interaction with nitrate. Nippon Yakurigaku Zasshi 1999; 114(4): 213- 8.

[76] Middendorff R, Davidoff MS, Behrends S, Mewe M, Miethens A, Muller D. Multiple roles of the messenger molecule cGMP in testicular function. Andrologia 2000; 32(1): 55-9.

[77] Rossato M, Di Virgilio F, Rizzuto R, Galeazzi C, Foresta C. Intracellular calcium store depletion and acrosome reaction in human spermatozoa: role of calcium and plasma membrance potential. Mol Hum Reprod 2001; 7(2): 119 -28.

[78] Tesarik J, Mendoza C, Carreras A. Effects of phosphodiesterase inhibitors caffeine and pentoxifylline on spontaneous and stimulus induced acrosome reactions in human sperm. Fertil Steril 1992; 58(6): 1185-90.

[79] Garbers DL, Lust DW, First NL, Lardy HA. Effects of phosphodiesterase inhibitors and cyclic nucleotides on sperm respiration and motility. Biochemistry 1971; 10: 1825-31.

[80] Yovich JL. Pentoxifylline: actions and applications in assisted reproduction. Hum Reprod 1993; 8(11): 1786- 91.

[81] Kovacic B, Vlaisavljevic V, Reljic M. Clinical use of pentoxifylline for activation of immotile testicular sperm before ICSI in patients with azoospermia. J Androl 2006; 27(1): 45-52.

[82] Tesarik J, Thébault A, Testart J. Effect of pentoxifylline on sperm movement characteristics in normozoospermic and asthenozoospermic specimens. Hum Reprod 1992; 7(9): 1257-63.

[83] Yunes R, Fernandez P, Doncel GF, Acosta AA. Cyclic nucleotide phosphodiesterase inhibition increases tyrosine phosphorylation and hyper motility in normal and pathological human spermatozoa. Biocell 2005; 29(3): 287-93.

[84] Freitas JP, Filipe PM. Pentoxifylline. A hydroxyl radical scavenger. Biol Trace Elem Res 1995; 47(1-3): 307-11.

[85] McKinney KA, Lewis SE, Thompson W. The effects of pentoxifylline on the generation of reactive oxygen species and lipid peroxidation in human spermatozoa. Andrologia 1996; 28(1): 15-20.

[86] Tournaye H, Janssens R, Verheyen G, Devroey P, Van SA. In vitro fertilization in couples with previous fertilization failure using sperm incubated with pentoxifylline and 2-deoxyadenosine. Fertil Steril 1994; 62(3): 574-9.

[87] Tesarik J, Mendoza C. Sperm treatment with pentoxifylline improves the fertilizing ability in patients with acrosome reaction insufficiency. Fertil Steril 1993; 60(1): 141-8.

[88] Negri P, Grechi E, Tomasi A, Fabbri E, Capuzzo A. Effectiveness of pentoxifylline in semen preparation for intrauterine insemination. Hum Reprod 1996; 11(6): 1236-9.

[89] Kay VJ, Coutts JR, Robertson L. Pentoxifylline stimulates hyperactivation in human spermatozoa. Hum Reprod 1993; 8(5): 727-31

[90] Lewis SE, Moohan JM, Thompson W. Effects of pentoxifylline on human sperm motility in normospermic individuals using computer-assisted analysis. Fertil Steril 1993; 59(2): 418-23.

[91] Sikka SC, Hellstrom WJ. The application of pentoxifylline in the stimulation of sperm motion is men undergoing electroejaculation. J Androl 1991; 12(3): 165-70.

[92] Moohan JL, Winston RLM, Lindsay KS. Variability of human sperm exposure to immediate and prolonged exposure to pentoxifylline. Hum Reprod 1993; 8(10): 1696-700.

[93] Centola GM, Cartie RJ, Cox C. Differential responses of human sperm to varying concentrations of pentoxyfylline with demonstration of toxicity. J Androl 1995; 16(2): 136-42. 
[94] Sharma RK, Tolentino MV Jr, Thomas AJ Jr, Agarwal A. Optimal dose and duration of exposure to artificial stimulants in cryopreserved human spermatozoa. J Urol 1996; 155(2): 568-73.

[95] Tasdemir M, Tasdemir I, Kodama H, Tanaka T. Pentoxifylline enhanced acrosome reaction correlates with fertilization in vitro. Hum Reprod 1993; 8(12): 2102-7.

[96] Kopf GS, Tubb DJ, Garbers DL. Activation of sperm respiration by a low molecular weight egg factor and by 8-bromoguanosine 3', 5'monophosphate. J Biol Chem 1979; 254(17): 8854-60.

[97] Glossmann H, Petrischor G, Bartsch G. Molecular mechanisms of the effects of sildenafil (VIAGRA). Exp Gerontol 1999; 34(3): 30518.

[98] Morales A, Gingell C, Collins M, Wicker PA, Osterloh IH. Clinical safety of oral sildenafil citrate (VIAGRA) in the treatment of erectile dysfunction. Int J Impot Res 1998; 10(2): 69-73.

[99] Aversa A, Mazzilli F, Rossi T, Delfino M, Isidori AM, Fabbri A. Effects of sildenafil (Viagra) administration on seminal parameters and post-ejaculatory refractory time in normal males. Hum Reprod 2000; 15(1): 131-4.

[100] Lefievre L, De Lamirande E, Gagnon C. The cyclic GMP-specific phosphodiesterase inhibitor, sildenafil, stimulates human sperm motility and capacitation but not acrosome reaction. J Androl 2000; 21(6): 929-37.

[101] Esteves SC, Glina S, Wonchockier R, Correa N. Sperm kinematics of normozoospermic specimens after stimulation by varying concentrations of a specific inhibitor of cGMP phosphodiesterase type-5 (Sildenafil) [abstract]. Fertil Steril 2003; 80(Suppl 3): 236.

[102] Esteves SC, Glina S, Verza Jr S, Wonchockier R, Correa N. Acrosome integrity and agonist-induced acrosome reaction rate after stimulation by varying concentrations of a specific inhibitor of cGMP phosphodiesterase type-5 (Sildenafil) in normozoospermic specimens [abstract]. Fertil Steril 2005; 84(Suppl 1): 225.

[103] Aitken RJ. The role of free oxygen radicals and sperm function. Int J Androl 1989; 12(2): 95-7.

[104] Liu DY, Baker HWG. The proportion of human sperm with poor morphology but normal intact acrosomes detected with Pisum sativum agglutinin correlates with fertilization in vitro. Fertil Steril 1988; 50(2): 288-93.

[105] Polanski FF, Lamb EJ. Do the results of semen analysis predict future fertility? A survival analysis study. Fertil Steril 1988; 49(6): 1059-65.
[106] Aitken RJ, Clarkson JS. Significance of reactive oxygen species in defining the efficacy of sperm preparation techniques. J Androl 1988; 9(6): 367-76.

[107] Barthelemy C, Royere D, Hammahah S, Lebos C, Tharanne MJ, Lansac J. Ultrastructural changes in membranes and acrosome of human sperm during cryopreservation. Arch Androl 1990; 25(1): 29-40.

[108] Aitken RJ, Best FS, Warner P, Templeton A. A prospective study of the relationship between semen quality and fertility in cases of unexplained infertility. J Androl 1984; 5: 297-303.

[109] Cooper TG, Noonan E, Eckardstein S, et al. World Health Organization reference values for human semen characteristics. Hum Reprod Update 2010; 16(3): 231-45.

[110] Katsuki T, Hara T, Ueda K, Tanaka J, Ohama K. Prediction of outcomes of assisted reproduction treatment using the calcium ionophore-induced acrosome reaction. Hum Reprod 2005; 20(2): 469-75.

[111] Whan LB, West M, McClure N, Lewis SEM. The effects of Delta9-tetrahydrocannabinol, the primary psychoactive cannabinoid in marijuana, on human sperm function in vitro. Fertil Steril 2006; 85(3): 653-60.

[112] Falzone N, Huyser C, Becker P, Leszczynski D, Franken DR. The effect of pulsed $900-\mathrm{MHz}$ GSM mobile phone radiation on the acrosome reaction, head morphometry and zona binding of human spermatozoa. Int $\mathrm{J}$ Androl 2010; 7. Available from: http:// onlinelibrary.wiley.com/doi/10.1111/j.1365-2605.2010.01054.x/ abstract [cited: $09^{\text {th }}$ Dec 2010].

[113] Mukhopadhyay D, Nandi P, Varghese AC, Gutgutia R, Banerjee S, Bhattacharyya AK. The in vitro effect of benzo[a]pyrene on human sperm hyperactivation and acrosome reaction. Fertil Steril 2010, 94(2): 595-8.

[114] Gupta SK, Chakravarty S, Suraj K, et al. Structural and functional attributes of zona pellucida glycoproteins [abstract]. Soc Reprod Fertil 2007; 63(Supp11): 203-16.

[115] Gupta SK, Bansal P, Ganguly A, Bhandari B, Chakrabarti K. Human zona pellucida glycoproteins: functional relevance during fertilization. J Reprod Immunol 2009; 83(1-2): 50-5.

[116] Chiu PC, Wong BS, Lee CL, et al. Zona pellucida-induced acrosome reaction in human spermatozoa is potentiated by glycodelin-A via down-regulation of extracellular signal-regulated kinases and up-regulation of zona pellucida-induced calcium influx. Hum Reprod 2010; 25(11): 2721-33.

This is an open access article licensed under the terms of the Creative Commons Attribution Non-Commercial License (http://creativecommons.org/licenses/by$\mathrm{nc} / 3.0 /$ ), which permits unrestricted, non-commercial use, distribution and reproduction in any medium, provided the work is properly cited. 